\title{
The role of Hippo signal pathway in breast cancer metastasis
}

This article was published in the following Dove Press journal:

OncoTargets and Therapy

\author{
Changran Wei' \\ Ying Wang ${ }^{2}$ \\ Xiangqi $\mathrm{Li}^{\mathrm{I}}$ \\ 'Department of Breast Surgery, \\ Affiliated Hospital of Taishan Medical \\ University, Tai'an, Shandong Province, \\ China; ${ }^{2}$ Rehabilitation Medicine, \\ Affiliated Hospital of Taishan Medical \\ University, Tai'an, Shandong \\ Province, China
}

Correspondence: Xiangqi Li

Department of Breast Surgery, Affiliated Hospital of Taishan Medical University, No 706 Taishan Road, Taishan District, Tai'an 271000, Shandong Province, China Email drlixqi@hotmail.com

\begin{abstract}
The Hippo pathway is a novel and highly conserved mammalian signaling pathway. Mutations and altered expression of core Hippo pathway components promote the migration, invasion, malignancy, and chemotherapy resistance of breast cancer cells. In cancer metastasis, tumor cells must detach from the primary tumor, invade surrounding tissue, and enter and survive in a foreign microenvironment. The metastatic potential of breast cancer is closely related to individual patient genetic profile. Nevertheless, the exact molecular mechanism that regulates the Hippo pathway in breast cancer metastasis is yet to be fully elucidated. This article discusses the function and regulation of the Hippo pathway, with focus given to its role in the context of breast cancer metastasis.
\end{abstract}

Keywords: breast cancer, Hippo signal pathway, metastasis

\section{Introduction}

In recent years, breast cancer has emerged as a major public health concern among women in our country. Each year, about 1.7 million women worldwide are diagnosed with breast cancer; over half of all cases (53\%) occur in less-developed regions. Breast cancer survival rate in high-income countries is higher than that in low- and middle-income countries. ${ }^{1}$ The incidence of breast cancer seems to be increasing, with more cases afflicting the younger patient population. The American Cancer Society (ACS) estimates that there will be 268,670 new cases of breast cancer diagnosed and 41,400 breast cancer deaths in $2018 .^{2}$ A significant proportion of cancer-associated deaths among breast cancer patients can be attributed to metastasis. Management of metastatic breast cancer is complex, and patient prognosis is often poor. ${ }^{3}$ Therefore, understanding the mechanisms that regulate metastasis and lymphatic angiogenesis are vital in order to develop efficacious cancer treatment modalities. ${ }^{4}$ Tumor biology and immunological studies of breast cancer have revealed complex processes involving multiple genetic regulatory mechanisms. The process of metastasis involves detachment of cancer cells from the primary tumor and invasion of these cells through widespread dissemination and requires metastatic cells to survive in a foreign microenvironment. Tumor metastatic potential is closely related to individual genetic profiles. ${ }^{5}$ Recent advancements in the field of oncology have led to the discovery of several complex and functionally diverse signaling pathways implicated in breast cancer metastasis. A common feature shared by these novel pathways are the involvement of cytokines. However, the underlying mechanisms of how these cytokines interact within the Hippo pathway as well as how the Hippo pathway interacts with other signaling pathways have not yet been fully clarified. It therefore comes as no surprise that the study of metastatic breast cancer remains a daunting task. 
The Hippo pathway has been found to regulate organ size and maintain tissue stability by governing cell proliferation and apoptosis. ${ }^{6}$ Mutations resulting in the altered expression of the main Hippo pathway components are associated with dysregulation in cell differentiation and organ development. ${ }^{7}$ Evidence surrounding the clinical implications of the Hippo pathway in breast cancer cases is scarce, and little is known about how the underlying molecular mechanisms of this pathway are regulated. In this review, we summarize the recent advances in understanding the functions of core components in the Hippo pathway with special attention given to its relationship with the clinical features of breast cancer metastasis.

\section{Organization and function of Hippo signaling pathway}

The study of Hippo signaling pathway began with the discovery of the Wts gene in Drosophila melanogaster in 1994 with the human homolog of the Wts gene discovered soon after. Subsequent studies in this field have revealed the complete structure of the Hippo pathway, including upstream active components, core kinase cascade, and downstream transcriptional effectors. ${ }^{8}$ The upstream active components comprise FAT4 (Fat homology), FEMD6 (Ex homology), Mer (NF2, Mer homology), and DCHS1/2 (Dachsous homology). The core kinase cascade consists of MST1/2 (Hpo orthologs), SAV1 (Sav orthologs), LATS1/2 (Wts orthologs), and Mob1 (Mats orthologs). The major effectors of the core component in the Hippo pathway is YAP (yes-associated protein, a yes-related protein) and TAZ (transcription co-activator with a PDZ binding motif, also called WWTR1; homologs of Yki). The transcription-related parts of the Hippo signaling pathway include TEF/TEAD1-4 (Sd orthologs), CTGF (connective tissue growth factor), AREG (amphiregulin), and Gli2.

In mammals, the core Hippo pathway comprises MST1/2, SAV1, LATS1/2, and Mob1 which are each activated by phosphorylation to form compounds that transmit apoptotic signals. ${ }^{9}$ MST1/2 and LATS1/2 are serine/tyrosine protein kinases, while SAV1 and Mob1 act as protein activators. YAP and TAZ are the primary transcription co-activators of the Hippo signaling pathway. Upstream regulators of the Hippo signaling pathway and the two core cascade molecules (LATS1 and MST1) have been found to function as tumor suppressor genes. The Hippo signaling pathway is a highly evolutionarily conserved pathway and functions mainly to regulate cell proliferation, tissue homoeostasis, and organ size. ${ }^{10-14}$ In normal physiological state, the pathway plays a central role in maintaining the balance between cell proliferation and apoptosis, its dysregulation is strongly associated with the occurrence and development of human tumor growth and carcinogenesis. ${ }^{15}$

\section{Regulatory mechanism of the HIPPO signaling pathway}

The Hippo signaling pathway is present ubiquitously in mammals, and its activity is strictly regulated. Hippo pathway activation is triggered by contact inhibition and substrateinduced stress that occur when cellular density reaches a certain threshold. ${ }^{16}$ The kinase cascade forms the core of the Hippo pathway and begins with MST1/2 phosphorylation of LATS1/2 to form a LATS1/2-MST1/2 complex, a process assisted by two scaffold proteins SAV1 and Mob1. ${ }^{17}$ Activated LATS1/2 then goes on to phosphorylate downstream effectors YAP and TAZ. YAP/TAZ proceeds to bind to a 14-3-3 protein which results in their cytoplasmic protease degradation. ${ }^{18}$ Phosphorylation of YAP/TAZ results in the inhibition of cell proliferation. These series of reactions are precisely regulated to control cell proliferation, apoptosis, and differentiation. ${ }^{19}$ Conversely, if the upstream kinases are not activated, the hypophosphorylated effector molecules YAP/TAZ translocate into the nucleus. ${ }^{20,21}$ Once in the nucleus, YAP/TAZ exerts their oncogenic functions by combining with the transcription factors TEA family (TEA domain family members, TEAD) of transcription factors to promote the expression of transcriptional genes involved in cell proliferation and epithelial-mesenchymal transition (EMT). ${ }^{14,16}$

Traditional understanding of Hippo pathway activation holds on to the view that upstream and downstream components interact in a direct manner. Later studies have since revealed the far more complex nature of the Hippo pathway, and MST1/2 has been found to not be the sole activator of LATS1/2. Moreover, MAP4K family members MAP4K1/2/3 and MAP4K4/6/7 have recently been identified to work in parallel with MST1/2 to activate LATS1/2. ${ }^{22-24}$ LATS1/2 phosphorylates the YAP S127 and TAZ S89 sites, creating binding sites for interactions with the 14-3-3 protein, leading to YAP/TAZ cytoplasm ubiquitylation and degradation, thereby nullifying their functions as transcription co-activators. ${ }^{18,19,22-24}$ Furthermore, GSK3 also phosphorylates TAZ and induces it to the $\mathrm{SCF}(\beta-\mathrm{TrCP}) \mathrm{E} 3$ ubiquitin ligase, leading to TAZ ubiquitylation and degradation. ${ }^{25}$ In contrast, when YAP and TAZ are not phosphorylated, they accumulate in the nucleus acting as transcriptional co-repressor genes that promote the expression of cell proliferation-related genes. ${ }^{26-28}$ 


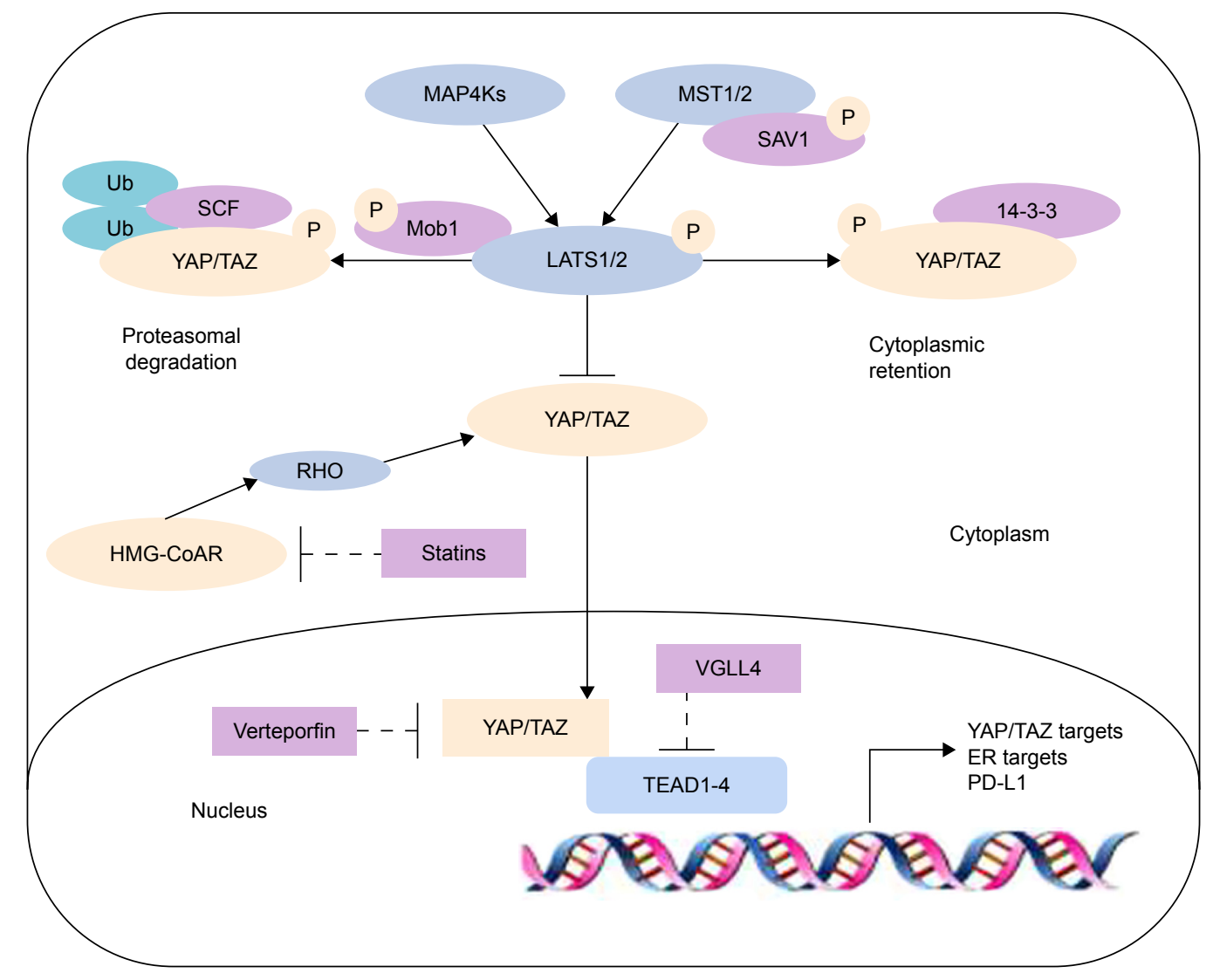

Figure I Regulation of the core components of the Hippo signaling pathway. Abbreviation: ER, estrogen receptor.

Therefore, the Hippo pathway is shown to negatively regulate the activity of YAP/TAZ by triggering a phosphorylation cascade (Figure 1). The Hippo pathway ultimately functions to regulate cell growth and inhibit tumorgenesis. ${ }^{15}$

\section{Crosstalk with other signaling pathways}

Extensive crosstalk between the Hippo pathway and other signaling pathways makes up extremely complex cellular signaling networks that collectively affect cell proliferation and apoptosis. Interactions with the classic Wnt pathway as well as mutations and increased stability of $\beta$-catenin have been found to be driving forces behind the formation of certain malignant tumors. The Hippo signaling pathway may antagonize WNT signaling pathway, leading to the direct combination of transcriptional co-activators YAP/TAZ and $\beta$-catenin, thereby effectively allowing the Hippo pathway to take on a significant role in regulating the nuclear location of $\beta$-catenin. ${ }^{29}$ In addition, the YAP-TEAD4-Smad3-p300 complex that arises due to Hippo pathway interactions with TGF pathways has been reported to augment the expression of CTGF and promote the proliferation of malignant mesothelioma cells and extracellular matrix deposition. ${ }^{30}$ Similarly, CTGF expression in hepatocellular carcinoma (HCC) cells seems to be dependent on crosstalk with the EGFR pathway. ${ }^{31}$ Therefore, the Hippo pathway has been found to have roles in various pathological processes involved in the development of breast cancer.

Recently, LATS has been discovered to directly interact with estrogen receptor- $\alpha(E R \alpha)$ signaling for ubiquitination of ER $\alpha$ that results in Ddb1-cullin4-associated-factor 1 (DCAF1)-dependent ER $\alpha$ ubiquitination and degradation. Lack of LATS will increase the stability of ER $\alpha$ and YAP/ TAZ, suggesting that the Hippo pathway may also be able to regulate the fate of breast cell. Moreover, decreased LATS levels in patients are associated with reduced relapse-free survival. Interestingly, this control of ER $\alpha$ is independent of YAP/TAZ. ${ }^{32}$ In addition, the expression of G-proteincoupled estrogen receptor (GPER) plays a critical role that mediates the Hippo/YAP/TAZ pathway in breast cancer. GPER has been found to be positively associated with TAZ expression in human invasive ductal carcinoma samples. ${ }^{33}$ HMG-CoA reductase is defined as a rate-limiting enzyme of the mevalonate pathway and correlates with the nuclear 
expression and transcriptional responses of YAP/TAZ. The mevalonate pathway is essential for the isoprenylation of small guanosine triphosphate (GTP)-binding proteins such as Ras and Rho. Inhibition of $\mathrm{HMG}-\mathrm{CoA}$ reductase prevents the growth and self-renewal of breast cancer cells through the decreased transcriptional activity of YAP/TAZ. ${ }^{34}$ PD-L1, an immune checkpoint molecule, has been recently identified as a transcriptional target of the Hippo signaling pathway. Studies have found that TAZ upregulates PD-L1 expression by binding to the PD-L1 promoter through the TEAD family of transcription factors, thereby disrupting the function of T cells. ${ }^{35}$ Exercise-induced catecholamines have been reported to be important for breast cancer cell development, including suppression of cell growth and tumorigenesis. Incubation with exercise-conditioned serum is able to induce phosphorylation of YAP in MCF-7 cells. Accordingly, total protein levels of YAP decreases through cytosolic sequestration. ${ }^{36}$ Functional studies identified glucocorticoids as hormonal activators target for YAP and increased YAP nuclear accumulation and transcriptional activity in MDA-MB-231. Glucocorticoid receptor (GR) activation was associated with bad prognosis in human breast cancer basal-like subtype. Targeting GR to prevent self-renewal of cancer stem cells and chemoresistance may provide a novel potential therapeutic. ${ }^{37}$

\section{Role of Hippo pathway components in breast cancer metastasis}

Breast cancer is one of the most common malignant tumors in women. As such, the molecular pathogenesis of breast cancer has been heavily scrutinized to obtain a deeper understanding of how to more effectively treat and manage this debilitating condition. A dysfunctional Hippo pathway promotes breast cancer metastasis through multiple mechanisms (Figure 2). The Hippo core components play a pivotal role in metastatic

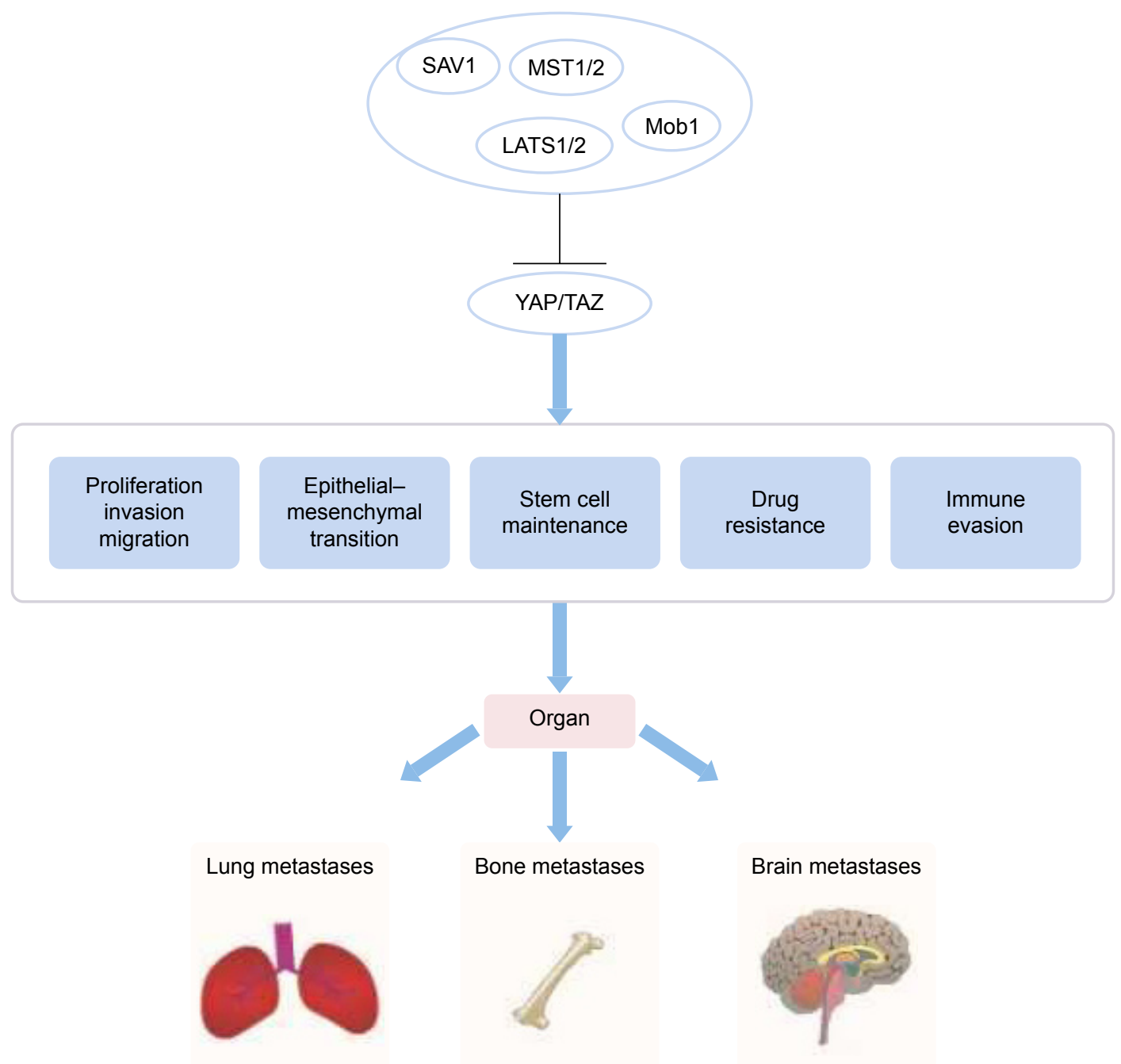

Figure 2 The Hippo pathway regulates organ metastases by controlling cell proliferation, EMT, and immune evasion. Abbreviation: EMT, epithelial-mesenchymal transition. 
breast tumor colonization in and outside breast tissue. ${ }^{38}$ YAP deficiency reduces the incidence of lung metastasis in a genetically engineered mouse model of breast cancer. The presence of Ski effectively reduces lung metastasis in TAZ-overexpressing breast cells..$^{39,40}$ The Hippo pathway also plays a role in breast cancer bone metastases, which occur in up to $70 \%$ of patients with advanced breast cancer. Phosphorylated HER3 Tyr1307 is able to methylate MST1 at the Lys59 site, leading to the activation of YAP/TAZ in tumor cells, which ultimately promotes bone metastasis. ${ }^{41}$ Bartucci et $\mathrm{al}^{42}$ found that TAZ nuclear expression in bone metastasis was significantly highly in comparison with its cytoplasmic expression in primary tumors. In addition, the hypoxic microenvironment present in bone marrow is highly conducive for tumor infiltration. Hypoxic states are characterized by the presence of hypoxia inducible factor (HIF)-1 $\alpha$. Bendinelli et al reported that HIF- $1 \alpha$ interacts with TAZ to stimulate breast cancer bone metastasis in a hypoxic microenvironment. ${ }^{43,44}$ Nuclear HIF-1 $\alpha$ has been found to be largely involved in the EMT in breast cancer metastasis, and this molecule has been demonstrated to be regulated by interactions between E-cadherin and Hippo pathway factors. ${ }^{45}$ Abelson tyrosine protein kinases (ABL) elevate the expression of both TAZ and activator of transcription 5 (STAT5) that together work to promote breast-to-bone metastasis. ${ }^{46}$ Aberrations in the Hippo signaling pathway also seem to confer increased chemoresistance to cancer cells. In vitro cultured breast cancer stem cells show increased chemoresistant properties and enhanced migratory potential when compared with differentiated, non-tumor breast cancer cells (dBCCs). Overexpression of TAZ in dBCCs induced neoplastic transformation and increased migratory activity. Conversely, the loss of TAZ in breast cancer stem cells severely impeded the formation of metastatic colonies and reduced chemoresistance. ${ }^{42}$ Overexpression of TAZ can increase resistance to taxols in the MCF10 breast cell line. On the other hand, reduced TAZ expression seems to downregulate the sensitivity of MDA-MB-231 breast cell line to taxols. ${ }^{47}$ Notably, decreased sensitivity of tamoxifen is associated with low YAP expression and is related to the clinical outcome in luminal A subgroup of breast cancer. ${ }^{48}$

Notably, the upstream kinases of the Hippo pathway, MST1/2 and LATS1/2, act as tumor suppressors that regulate YAP phosphorylation. ${ }^{49,50}$ Further studies have found that the decreased expression of LATS1 or LATS2 mRNA in breast cancer tissues leads to increased tumor size and lymph node metastasis and is negatively related to the presence of estrogen and progesterone receptors. ${ }^{51}$ Several lines of evidence suggest that the deletion or mutation of LATS $1 / 2$ leads to a more aggressive breast cancer phenotype that shows enhanced invasive ability. Taken together, these studies present convincing evidence that LATS1/2 may be a novel target for anticancer treatment in breast cancer. ${ }^{52}$ However, less is known about the role of MST in breast cancer metastasis. Existing studies suggest that high MST1 expression in MCF-7 breast cancer cells can inhibit cell proliferation and promote cell apoptosis. ${ }^{53}$ Therefore, MST1 is also considered to be a tumor suppressor.

Interestingly, studies allude toward the oncogenic functions of YAP. The potential carcinogenicity of YAP is related to its hypophosphorylated state, which can increase the expression of growth factors and apoptosis-inhibiting factors. Inhibition of Hippo signaling results in YAP hypophosphorylation and its nuclear localization. In the nucleus, YAP binds to transcription factors to increase the expression of cyclin D1. However, cyclin E expression does not change. Overexpression of YAP in MCF10A breast cell line can induce EMT, which is associated with the upregulation of fibronectin, vimentin, and N-cadherin and the downregulation of E-cadherin and occludin. The activation of AKT and ERK increases the ability of YAP to promote the proliferation of MCF10A cells in the absence of EGF ${ }^{54}$ Cells grown at high density were less effective compared with low cell density at invading an endothelial cell monolayer. Treatment with verteporfin and YAP knockdown induced downregulation of the cytokines IL6, IL8, and CXC1, CXC2, and CXC3 by inhibiting the activity of YAP and consequently inhibited vascular invasiveness of breast cancer cells. ${ }^{55}$ These data suggest that altered growth density of breast cancer cells regulates their vascular invasion by LATS1-YAP in the Hippo pathway. In addition, TAZ expression is positively associated with EMT, tumorigenesis, tumor migration, and invasion in breast cancer cells. ${ }^{56,57} \mathrm{TAZ}$ is highly expressed in Hs578T, BT-549, and MDA-MB-435S invasive breast cancer cell lines and moderately expressed in MDA-MB-231 cells. ${ }^{58}$ Wang et al found that Twist upregulated the expression of PAR1, would inhibit TAZ phosphorylation, and increases TAZ activity. TAZ depletion suppressed transendothelial invasion of T47D breast cancer cells. ${ }^{59}$

In contrast to its oncogenic activity of YAP/TAZ in the development of breast cancer and tumorigenesis, there is a school of thought that believes that YAP/TAZ may also possess crucial tumor suppressive role in human oncogenesis. ${ }^{60}$ Yuan et $\mathrm{a}^{61}$ reported that immunohistochemical assay of breast tissues revealed significant nuclear YAP expression in normal breast tissues, with YAP expression being low or 
even absent in cancer tissues. Similar findings were reported by Jaramillo-Rodriguez et al. ${ }^{62}$ YAP expression is weak in luminal epithelial cells. Knockdown of YAP promotes cell migration, invasion, and tumor growth. ${ }^{61}$ Similarly, Tufail et al suggested that reduced expression of YAP is negatively associated with the presence of estrogen and progesterone receptors in invasive breast carcinomas. ${ }^{63}$ YAP1 interacts with the p53 family member p 73 to mediate the nuclear stability of p73, which leads to the expression of apoptotic genes. ${ }^{64}$

In addition, research examining the relationship between the expression of Hippo pathway factors and clinical outcomes of breast cancer hints toward their potential to function as predictive clinical biomarkers in breast cancer patients. Immunohistochemistry investigations in 69 breast cancer samples revealed that $75.4 \%$ of clinical breast cancer samples demonstrated elevated YAP expressions, with $29 \%$ of these cases showing high expressions of YAP. However, $24.6 \%$ of these breast cancer samples demonstrated no YAP expression. It has been suggested that overexpression of YAP correlates with tumor formation and growth. ${ }^{65}$ Immunohistochemistry analysis of TAZ expression in 640 invasive breast carcinoma samples that comprised estrogen and progesterone receptorpositive (ER+/PR+), HER2-positive and triple-negative (TN) tumors suggested that TAZ expression was significantly associated with the TN phenotype (60.5\% TAZ-positive, $P<0.001)$. Similarly, other studies have reported high TAZ expression in the HER2-positive, luminal B, and TN breast cancer. ${ }^{42,66-69}$ TAZ was highly expressed in $90 \%$ of breast carcinomas with EMT. An increased proportion of patients whose tumors strongly expressed TAZ experienced higher rates of tumor recurrence and worse survival outcomes. YAP/TAZ acts as an oncogene in different subtypes of breast cancer. ${ }^{70}$ The risk of residual disease in HER2-positive or TN breast cancer patients after receiving neoadjuvant therapy has been shown to be related to the subcellular location of both pMST1/2 and TAZ. Patients with strong TAZ and MST1/2 nuclear expressions showed poorer clinical outcomes after neoadjuvant therapy. Conversely, the colocalization of cytoplasmic pMST1/2 and TAZ is likely to be a protective factor in the Hippo pathway. Therefore, MST1/2 may function as a potential predictive biomarker in HER2positive and TN breast cancer patients. ${ }^{71}$ The expression of TAZ/YAP was associated with inferior survival in male breast cancer patients. ${ }^{72}$ The above studies support the notion that TAZ/YAP expression is correlated with tumor metastasis and patient outcomes. TAZ/YAP has been found to regulate cell proliferation, migration, metastasis, EMT, resistance to chemotherapy, and CSCs at the preclinical research level.

\section{Future challenges and conclusion}

The critical role of core components in Hippo signaling is supported by the overwhelming preclinical research and clinical analysis in the development of breast cancer. Both YAP and TAZ promote breast cancer cell proliferation, EMT, tumor growth, and drug resistance. The expression of YAP/ TAZ was also linked to patients' survival outcomes. Thus, the components of the Hippo pathway can serve as predictive biomarkers and as therapeutic targets for the treatment and management of breast cancer.

Verteporfin is an antagonist of YAP-TEAD transcriptional activity by competitively binding to YAP and abrogating the interaction of YAP with TEAD. Several studies found that verteporfin downregulated the transcriptional activity of YAP, which, in turn, inhibited the YAP-induced breast tumorigenesis. ${ }^{73,74}$ In addition, VGLL4 was recently identified as an inhibitor of YAP/TAZ-TEAD complex by competing with YAP/TAZ for TEAD binding. ${ }^{75}$ Statins have been used to target HMG-CoA reductase and inhibit the activity of Rho GTPases. Treatment with statins induces the inhibition of YAP and TAZ by increasing the activity of LATS $1 / 2$ and consequently inhibiting breast cancer cell migration and invasion. ${ }^{34}$ In a recent work by Moroishi et al, it has been found that the loss of LATS1/2 in breast cancer cells inhibits breast tumor growth by enhancing antitumor immune responses, suggesting that targeting LATS1/2 is an attractive strategy for cancer immunotherapy. ${ }^{76}$ Kinase inhibitors would be an exciting therapeutic approach for breast cancer. However, pharmacological studies for TAZ/YAP inhibition and the complex mechanism of LATS1/2 inhibition are still lacking. Thus, it may be challenging to explore the therapeutic potentials of the Hippo pathway in breast cancer. TAZ and PD-L1 are highly expressed in TN breast cancers. ${ }^{77}$ Furthermore, human anti-PD-L1 IgG1 antibody was applied to TN breast cancer patients in clinical trials. The investigation is still ongoing. ${ }^{78}$ There is an urgent need to develop an inhibitor of TAZ for immunotherapy. It is noteworthy that targeting the Hippo pathway components for breast cancer treatment has important clinical implications. ${ }^{79}$

The relationship between YAP/TAZ expression and different breast cancer subtypes still remain unclear, with different subtypes exhibiting varying YAP/TAZ expression levels. It has been suggested that YAP is able to function as either an oncogenic cancer gene or a tumor suppressor gene depending on the specific internal environment that is dictated by different subtypes of breast cancer. Several upstream components and downstream target genes of Hippo pathway are also intricately involved in the pathogenic process of breast 
cancer metastasis. Nevertheless, more research is necessary to further identify factors that remain unknown. In addition, Hippo crosstalk with other cellular signaling pathways which can reveal important information on the overall regulation of breast cancer tumorigenesis and metastasis is not well understood and would benefit from more in-depth research.

In conclusion, the Hippo pathway plays an undeniably important role in breast cancer, based on converging evidence from both clinical and experimental investigations. The future of breast cancer metastasis management would benefit tremendously from additional scrutinization of the Hippo pathway, which will pave the way for the development of more accurate prognosticating markers and treatment targets.

\section{Acknowledgment}

This work was supported by the following projects: the National Natural Science Foundation of China (No 81473687), Shandong Provincial Natural Science Foundation, China (No ZR2009CM039 and No ZR2013HM038), Health Science and Technology Plan of Shandong Health Science Association (No 2016BJ0015), and Tai'an Science and Technology Plan (No 2015NS2082).

\section{Disclosure}

The authors report no conflicts of interest in this work.

\section{References}

1. Altobelli E, Rapacchietta L, Angeletti P, et al. Breast cancer screening programmes across the WHO European region: differences among countries based on national income level. Int J Environ Res Public Health. 2017;14(4):E452.

2. Siegel RL, Miller KD, Jemal A. Cancer statistics, 2018. CA Cancer J Clin. 2018;68(1):7-30.

3. Ginsburg O, Bray F, Coleman MP, et al. The global burden of women's cancers: a grand challenge in global health. Lancet. 2016; 389(10071):847-860.

4. Li X, Dang X, Sun X. Expression of survivin and VEGF-C in breast cancer tissue and its relation to lymphatic metastasis. Eur J Gynaecol Oncol. 2012;33(2):178-182.

5. Talmadge JE, Fidler IJ. AACR centennial series: the biology of cancer metastasis: historical perspective. Cancer Res. 2010;70(14): 5649-5669.

6. Fu V, Plouffe SW, Guan KL. The Hippo pathway in organ development, homeostasis, and regeneration. Curr Opin Cell Biol. 2018;49: 99-107.

7. Meng Z, Moroishi T, Guan KL. Mechanisms of Hippo pathway regulation. Genes Dev. 2016;30(1):1-17.

8. Justice RW, Zilian O, Woods DF, Noll M, Bryant PJ. The Drosophila tumor suppressor gene warts encodes a homolog of human myotonic dystrophy kinase and is required for the control of cell shape and proliferation. Genes Dev. 1995;9(5):534-546.

9. Zhao B, Li L, Guan KL. Hippo signalling at a glance. Cell Sci. 2010; 123(23):4001-4006.

10. Barry ER, Camargo FD. The Hippo superhighway: signalling cross roads converging on the Hippo/Yap pathway in stem cells and development. Curr Opin Cell Biol. 2013;25:247-253.
11. Varelas X. The Hippo pathway effectors TAZ and YAP in development, homeostasis and disease. Development. 2014;141(8):1614-1626.

12. Yu FX, Meng Z, Plouffe SW, Guan KL. Hippo pathway regulation of gastrointestinal tissues. Annu Rev Physiol. 2014;77:201-227.

13. Moroishi T, Hansen CG, Guan KL. The emerging roles of YAP and TAZ in cancer. Nat Rev Cancer. 2015;15(2):73-79.

14. Piccolo S, Dupont S, Cordenonsi M. The biology of YAP/TAZ: hippo signalling and beyond. Physiol Rev. 2014;94(4):1287-1312.

15. Atkins M, Potier D, Romanelli L, et al. An ectopic network of transcription factors regulated by hippo signaling drives growth and invasion of a malignant tumor model. Curr Biol. 2016;26(16):2101-2113.

16. Zhao B, Wei XM, Li WQ, et al. Inactivation of YAP oncoprotein by the Hippo pathway is involved in cell contact inhibition and tissue growth control. Genes Dev. 2007;21(21):2747-2761.

17. Harvey K, Tapon N. The Salvador-Warts-Hippo pathway an emerging tumour-suppressor network. Nat Rev Cancer. 2007;7(3):182-191.

18. Zhao B, Li L, Lei QY, Guan KL. The Hippo-YAP pathway in organ size control and tumorigenesis: an updated version. Genes Dev. 2010; 24(9):862-874.

19. Hoa L, Kulaberoglu Y, Gundogdu R, et al. The characterisation of LATS2 kinase regulation in Hippo-YAP signalling. Cell Signal. 2016; 28(5):488-497.

20. Liu CY, Lv XB, Li TT, et al. PP1 cooperates with ASPP2 to dephosphorylate and activate TAZ. J Biol Chem. 2011;286(7): 5558-5566.

21. Yu FX, Zhao B, Guan KL. Hippo pathway in organ size control, tissue homeostasis, and cancer. Cell. 2015;163(4):811-828.

22. Li Q, Li SX, Mana-Capelli S, et al. The conserved misshapen-wartsYorkie pathway acts in enteroblasts to regulate intestinal stem cells in Drosophila. Dev Cell. 2014;31(3):291-304.

23. Meng ZP, Moroishi T, Mottier-Pavie V, et al. MAP4K family kinases act in parallel to MST1/2 to activate LATS1/2 in the Hippo pathway. Nat Commun. 2015;6:8357.

24. Zheng YG, Wang W, Liu B, Deng H, Uster E, Pan D. Identification of happyhour/MAP4K as alternative $\mathrm{Hpo} / \mathrm{Mst}-$ like kinases in the hippo kinase cascade. Dev Cell. 2015;34(6):642-655.

25. Huang W, Lv XB, Liu CY, et al. The N-terminal phosphodegron targets TAZ/WWTR1 protein for SCF $\beta$-TrCP-dependent degradation in response to phosphatidylinositol 3-kinase inhibition. J Biol Chem. 2012;287(31):26245-26253.

26. Lei QY, Zhang H, Zhao B, et al. TAZ promotes cell proliferation and epithelial-mesenchymal transition and is inhibited by the hippo pathway. Mol Cell Biol. 2008;28(7):2426-2436.

27. Schlegelmilch K, Mohseni M, Kirak O, et al. YAP1 acts downstream of $\alpha$-catenin to control epidermal proliferation. Cell. 2011;144(5): 782-795.

28. Kim M, Kim T, Johnson RL, Lim DS. Transcriptional co-repressor function of the hippo pathway transducers YAP and TAZ. Cell Rep. 2015;11(2):270-282.

29. Imajo M, Miyatake K, Iimura A, Miyamoto A, Nishida E. A molecular mechanism that links Hippo signalling to the inhibition of Wnt/betacatenin signalling. EMBO J. 2012;31(5):1109-1122.

30. Fujii M, Toyoda T, Nakanishi H, et al. TGF- $\beta$ synergizes with defects in the Hippo pathway to stimulate human malignant mesothelioma growth. J Exp Med. 2012;209(3):479-494.

31. Urtasun R, Latasa MU, Demartis MI, et al. Connective tissue growth factor autocriny in human hepatocellular carcinoma: oncogenic role and regulation by epidermal growth factor receptor/yes-associated protein-mediated activation. Hepatology. 2011;54(6):2149-2158.

32. Britschgi A, Duss S, Kim S, et al. The Hippo kinases LATS1 and 2 control human breast cell fate via crosstalk with ER $\alpha$. Nature. 2017; 541(7638):541-545.

33. Zhou X, Wang SY, Wang Z, et al. Estrogen regulates Hippo signalling via GPERin breast cancer. Clin Invest. 2015;125(5):2123-2135.

34. Sorrentino G, Ruggeri N, Specchia V, et al. Metabolic control of YAP and TAZ by the mevalonate pathway. Nat Cell Biol. 2014;16(4): $357-366$. 
35. Van Rensberg HJJ, Azad T, Ling M, et al. The Hippo pathway component TAZ promotes immune evasion in human cancer through PD-L1. Cancer Res. 2018;78(6):1457-1470.

36. Dethlefsen C, Hansen LS, Lillelund C, et al. Exercise-induced catecholamines activate the Hippo tumor suppressor pathway to reduce risks of breast cancer development. Cancer Res. 2017;77(18): 4894-4904.

37. Sorrentino G, Ruggeri N, Zannini A, et al. Glucocorticoid receptor signalling activates YAP in breast cancer. Nat Commun. 2017;8:14073.

38. Bos PD, Zhang XHF, Nadal C, et al. Genes that mediate breast cancer metastasis to the brain. Nature. 2009;459(7249):1005-1009.

39. Chen Q, Zhang N, Gray RS, et al. A temporal requirement for Hippo signalling in mammary gland differentiation, growth and tumorigenesis. Genes Dev. 2014;28(5):432-437.

40. Rashidian J, Le Scolan E, Ji X, et al. Ski regulates Hippo and TAZ signalling to suppress breast cancer progression. Sci Signal. 2015; 8(363):ra14.

41. Li C, Wang S, Xing Z, et al. A ROR1-HER3-lncRNA signalling axis modulates the Hippo-YAP pathway to regulate bone metastasis. Nat Cell Biol. 2017;19(2):106-119.

42. Bartucci M, Dattilo R, Moriconi C, et al. TAZ is required for metastatic activity and chemoresistance of breast cancer stem cells. Oncogene. 2015;34(6):681-690.

43. Xiang L, Gilkes DM, Hu H, et al. HIF- $1 \alpha$ and TAZ serve as reciprocal co-activators in human breast cancer cells. Oncotarget. 2015;6(14): $11768-11778$

44. Bendinelli P, Maroni P, Matteucci E, et al. Hypoxia inducible factor-1 is activated by transcriptional co-activator with PDZ-binding motif(TAZ) versus WW domain-containing oxidoreductase (WWOX) in hypoxic microenvironment of bone metastasis from breast cancer. Eur J Cancer. 2013;49(11):2608-2618.

45. Maroni P, Matteucci E, Drago L, et al. Hypoxia induced E-cadherin involving regulators of Hippo pathway due to HIF-1 stabilization/ nuclear translocation in bone metastasis from breast carcinoma. Exp Cell Res. 2015;330(2):287-299.

46. Wang J, Rouse C, Jasper JS, Pendergast AM. ABL kinases promote breast cancer osteolytic metastasis by modulating tumor-bone interactions through TAZ and STAT5 signalling. Sci Signal. 2016; 9(413):ra12

47. Lai D, Ho KC, Hao Y, Yang X. Taxol resistance in breast cancer cells is mediated by the hippo pathway component TAZ and its downstream transcriptional targets Cyr61 and CTGF. Cancer Res. 2011;71(7): $2728-2738$.

48. Lehn S, Tobin NP, Sims AH, et al. Decreased expression of YAP is associated with outcome in the luminal A breast cancer subgroup and with an impaired tamoxifen response. BMC Cancer. 2014;14:119.

49. Heidary Arash E, Shiban A, Song S, Attisano L. MARK4 inhibits Hippo signalling to promote proliferation and migration of breast cancer cells. EMBO Rep. 2017;18(3):420-436.

50. Hay BA, Guo M. Coupling cell growth, proliferation, and death. Hippo weighs in. Dev Cell. 2003;5(3):361-363.

51. Visser S, Yang X. LATS tumor suppressor: a new governor of cellular homeostasis. Cell Cycle. 2010;9(19):3892-3903.

52. Cooper J, Giancotti FG. Cancer: a new role for non-canonical Hippo signalling. Cell Res. 2017;27(4):459-460.

53. Luo X-L, Li Z-M, Yan Q, et al. Effects of MST1 on cell proliferation and apoptosis of human breast carcinoma cell line MCF-7. Tumor. 2008;28(10):852-854.

54. Overholtzer M, Zhang JM, Smolen GA, et al. Transforming properties of YAP, a candidate oncogene on the chromosome 11q22 amplicon. Proc Natl Acad Sci U S A. 2006;103(33):12405-12410.

55. Sharif GM, Schmidt MO, Yi C, et al. Cell growth density modulates cancer cell vascular invasion via Hippo pathway activity and CXCR2 signaling. Oncogene. 2015;34(48):5879-5889.

56. Lei QY, Zhang H, Zhao B, et al. TAZ promotes cell proliferation and epithelial mesenchymal transition and is inhibited by the hippo pathway. Mol Cell Biol. 2008;28(7):2426-2436.
57. Mi W, Lin Q, Childress C, et al. Geranylgeranylation signals to the Hippo pathway for breast cancer cell proliferation and migration. Oncogene. 2015;34(24):3095-3106.

58. Chan SW, Lim CJ, Guo K, et al. A role for TAZ in migration, invasion and tumorigenesis of breast cancer cells. Cancer Res. 2008;68(8): 2592-2598.

59. Wang Y, Liu J, Ying X, Lin PC, Zhou BP. Twist-mediated epithelialmesenchymal transition promotes breast tumor cell invasion via inhibition of Hippo pathway. Sci Rep. 2016;6:24606.

60. Wang H, Du YC, Zhou XJ, Liu H, Tang SC. The dual functions of YAP-1 to promote and inhibit cell growth in human malignancy. Cancer Metastasis Rev. 2014;33(1):173-182.

61. Yuan M, Tomlinson V, Lara R, et al. Yes-associated protein (YAP) functions as a tumor suppressor in breast. Cell Death Differ. 2008; 15(11):1752-1759.

62. Jaramillo-Rodriguez Y, Cerda-Flores RM, Ruiz-Ramos R, et al. YAP expression in normal and neoplastic breast tissue: an immunohistochemical study. Arch Med Res. 2014;45(3):223-228.

63. Tufail R, Jorda M, Zhao W, Reis I, Nawaz Z. Loss of yes-associated protein (YAP) expression is associated with estrogen and progesterone receptors negativity in invasive breast carcinomas. Breast Cancer Res Treat. 2012;31(3):743-750.

64. Matallanas D, Romano D, Yee K, et al. RASSF1A elicits apoptosis through an MST2 pathway directing proapoptotic transcription by the p73 tumor suppressor protein. Mol Cell. 2007;27(6):962-975.

65. Wang XD, Su L, Ou Q. Yes-associated protein promotes tumour development in luminal epithelial derived breast cancer. Eur J Cancer. 2012;48(8):1227-1234.

66. Kim SK, Jung WH, Koo JS. Yes-associated protein (YAP) is differentially expressed in tumor and stroma according to the molecular subtype of breast cancer. Int J Clin Exp Pathol. 2014;7:3224-3234.

67. Vici P, Mottolese M, Pizzuti L, et al. The Hippo transducer TAZ as a biomarker of pathological complete response in HER2-positive breast cancer patients treated with trastuzumab-based neoadjuvant therapy. Oncotarget. 2014;5(20):9619-9625.

68. Lehn S, Tobin NP, Sims AH, et al. Decreased expression of yes-associated protein is associated with outcome in the luminal A breast cancer subgroup and with an impaired tamoxifen response. BMC Cancer. 2014;14:119.

69. Min Kim H, Kim SK, Jung WH, Koo JS. Metaplastic carcinoma show different expression pattern of YAP compared to triple-negative breast cancer. Tumor Biol. 2014;36(2):1207-1212.

70. Díaz-Martín J, López-García MÁ, Romero-Pérez L, et al. Nuclear TAZ expression associateswith the triple-negative phenotype in breast cancer. Endocr Relat Cancer. 2015;22(3):443-454.

71. Ercolani C, Di Benedetto A, Terrenato I, et al. Expression of phosphorylated Hippo pathway kinases (MST1/2 and LATS1/2) in HER2-positive and triple-negative breast cancer patients treated with neoadjuvant therapy. Cancer Biol Ther. 2017;18(5):339-346.

72. Di Benedetto A, Mottolese M, Sperati F, et al. Association between AXL, Hippo transducers, and survival outcomes in male breast cancer. J Cell Physiol. 2017;232(8):2246-2252.

73. Wang C, Zhu X, Feng W, et al. Verteporfin inhibits YAP function through up-regulating 14-3-3 $\sigma$ sequestering YAP in the cytoplasm. Am J Cancer Res. 2015;6(1):27-37.

74. Oku Y, Nishiya N, Shito T, et al. Small molecules inhibiting the nuclear localization of YAP/TAZ for chemotherapeutics and chemosensitizers against breast cancers. FEBS Open Bio. 2015;5(1):542-549.

75. Jiao $\mathrm{S}$, Wang $\mathrm{H}$, Shi Z, et al. A peptide mimicking VGLL4 function acts as a YAP antagonist therapy against gastric cancer. Cancer Cell. 2014; 25(2):166-180.

76. Moroishi T, Hayashi T, Pan WW, et al. The Hippo Pathway Kinases LATS1/2 Suppress Cancer Immunity. Cell. 2016;167(6): 1525.e17-1539.e17.

77. Mittendorf EA, Philips AV, Mericbernstam F, et al. PD-L1 Expression in Triple-Negative Breast Cancer. Cancer Immunol Res. 2014; 2(4):361-370. 
78. Schmid P, Cruz C, Braiteh FS, et al. Abstract 2986: atezolizumab in metastatic TNBC (mTNBC): long-term clinical outcomes and biomarker analyses. Cancer Res. 2017;77(13 Suppl):2986-2986.
79. Ye S, Eisinger-Mathason TS. Targeting the Hippo pathway: clinical implications and therapeutics. Pharmacol Res. 2015;103:270-278.

\section{Publish your work in this journal}

OncoTargets and Therapy is an international, peer-reviewed, open access journal focusing on the pathological basis of all cancers, potential targets for therapy and treatment protocols employed to improve the management of cancer patients. The journal also focuses on the impact of management programs and new therapeutic agents and protocols on

\section{Dovepress}

patient perspectives such as quality of life, adherence and satisfaction. The manuscript management system is completely online and includes a very quick and fair peer-review system, which is all easy to use. Visit http://www.dovepress.com/testimonials.php to read real quotes from published authors.

Submit your manuscript here: http://www.dovepress.com/oncotargets-and-therapy-journal 